\title{
On the locus of the semantic satiation effect: Evidence from event-related brain potentials
}

\author{
JOHN KOUNIOS \\ University of Pennsylvania, Philadelphia, Pennsylvania \\ SONJA A. KOTZ \\ Max Planck Institute of Cognitive Neuroscience, Leipzig, Germany \\ and \\ PHILLIP J. HOLCOMB \\ Tufts University, Medford, Massachusetts
}

\begin{abstract}
The present study sought to determine whether semantic satiation is merely a by-product of adaptation or satiation of upstream, nonsemantic perceptual processes or whether the effect can have a locus in semantic memory. This was done by measuring event-related brain potentials (ERPs) in a semantic word-detection task involving multiple presentations of primes and critical related and unrelated words in three experiments involving visual (Experiment 1) and auditory (Experiments $2 \mathrm{~A}$ and 2B) stimuli. Primes varied in their type case (Experiment 1) or pitch (Experiment 2B) in order to discourage sensory adaptation. Prime satiation and relatedness of the primes to the critical word had interacting effects on ERP amplitude to critical words, particularly within the time-window of the N400 component. Because numerous studies have indicated a role for the $\mathrm{N} 400$ in semantic processing, modulation of the N400 relatedness effect by prime satiation (with little or no contribution from perceptual adaptation) suggests that semantic memory can be directly satiated, rather than the cost to semantic processing necessarily resulting from impoverishment of perceptual inputs.
\end{abstract}

When a word is repeatedly produced or perceived, many people experience what has become known as the semantic satiation effect, a subjective and temporary loss of the meaning of that word. Early research by adherents of the introspectionist school analyzed the phenomenal qualities of semantic satiation (e.g., Severance \& Washburn, 1907). More recent research has shown that such repetitive priming inhibits performance in semantic verification tasks (Smith, 1984; Smith \& Klein, 1990).

\section{RECENT STUDIES}

Three significant recent studies have sought to answer important questions concerning the locus and nature of the semantic satiation effect. Balota and Black (1997) examined semantic satiation in younger and older adults in a series of semantic verification experiments. Their basic paradigm required participants to make speeded judgments about whether two visually presented words were

Preparation of this report was supported by NIMH Grant MH57501 to J.K. and NICHD Grant HD25889 to P.J.H. Correspondence concerning this article may be sent to J. Kounios at the Institute for Research in Cognitive Science, University of Pennsylvania, 3401 Walnut Street, Suite 400A, Philadelphia, PA 19104-6228 (e-mail: jkounios@) psych.upenn.edu), or to P. J. Holcomb, Department of Psychology, Tufts University, Medford, MA 02155 (e-mail: pholcomb@tufts. edu). semantically related (e.g., royalty-queen) or not (e.g., royalty-box) after 2,12 , or 22 visual presentations of one of the members of the pair. They found evidence of semantic satiation, defined as a diminution of the difference in mean reaction time and accuracy between responses to related and unrelated pairs. Specifically, they reported that (1) the relatedness effect decreased with more repetition in younger participants (particularly for related pairs of relatively low strength of association-e.g., royalty$d u k e$ ), whereas older participants did not yield reliable evidence of such satiation; (2) such satiation did not depend on overt pronunciation of the satiated item (Experiment 2); (3) satiation did not occur in a phonological task requiring rhyming judgments (Experiment 3 ), implying that the effect was truly semantic and not sensory or perceptual in nature; and (4) satiation was mediated by semantic relatedness of specific items and not by general satiation of semantic memory. In sum, the results of Balota and Black suggest that semantic satiation is truly semantic and that such satiation may reflect some type of inhibitory process that diminishes with age.

There is, however, at least one significant methodological concern about the experiments reported by Balota and Black (1997) that render their results somewhat uncertain. Specifically, they operationalized semantic satiation as the difference in mean reaction times (and accuracies) between responses to related and unrelated word pairs, with a decrease in this difference implying satia- 
tion. Unfortunately, this is a somewhat ambiguous metric of satiation, because related and unrelated pairs required different judgments (i.e., related vs. unrelated), rendering a direct comparison of the corresponding reaction times somewhat problematic (e.g., due to a possible differential influence of response bias). So, the results of Balota and Black, although tantalizing, are not definitive.

In another recent study, Pilotti, Antrobus, and Duff (1997) examined the issue of whether the primary effect of semantic satiation is really on semantic information processing or whether the primary effect is on functionally prior sensory processes. Their idea was that a decrement in the efficiency of the processing of the meaning of an auditorially presented word could result from satiation, inhibition, or adaptation of an acoustic system whose outputs feed into a semantic system. In the latter case, if the inputs to the semantic system are of relatively poor quality, the outputs of the semantic system might be poorer as well

Pilotti et al. (1997) tested this hypothesis by having participants listen to words on a series of trials, with each trial consisting of either 3 or 30 presentations of a category prime (e.g., flower), followed by a pair of exemplar terms drawn from either the prime category (e.g., rose-tulip) or another category (e.g., shrimp-lobster). The participants indicated by buttonpress whether the words in the target pair were drawn from the same or different categories. The critical manipulation was intended to influence satiation of sensory acoustic codes required for word recognition, specifically whether the presentations of the category prime on a given trial were all in the same voice or whether they were in different voices. In fact, when all the presentations of the prime were in the same voice, they found evidence of satiation. Specifically, they found that massed prime repetition slowed responses to word pairs drawn from the same category as the prime, relative to responses to word pairs drawn from a category different from the prime, but only when all the primes were presented in the same voice (i.e., there was no satiation when the primes were presented in different voices).

Like the results of Balota and Black (1997), the results of Pilotti et al. (1997) are suggestive, but not definitive. One concern in the multiple-speaker condition is that switches among different voices might have induced the participants to pay more attention to the acoustic properties of the primes than to their meanings. Such a diversion of attention may have diminished semantic priming in the multiple-speaker condition. Furthermore, this tendency might have been exacerbated by the experimenters' use of a between-subjects design in which the two groups could have adopted differential strategies for the singleand multiple-speaker conditions (e.g., attending to the meaning of the primes or attending to the sound of the primes). Moreover, the results of Pilotti et al. contradict those of Balota and Black (Experiment 3), who found no evidence of phonological satiation in a rhyme-verification task, although the phonological codes tapped by the Balota and Black task may have been different from the acoustic codes presumably satiated in the Pilotti et al. experiment.

The third recent study, by Frenck-Mestre, Besson, and Pynte (1997), utilized a different approach to studying semantic satiation. Instead of measuring the effects of massed word repetition on such measures of performance as mean reaction time and accuracy, they looked for neurophysiological correlates of satiation. Specifically, they measured event-related brain potentials (ERPs) during a satiation task.

ERPs are obtained by measuring a participant's electroencephalogram (EEG), using electrodes attached to the scalp. The EEG can be measured while the subject performs a task involving the occurrence of discrete events, such as stimuli or responses. Segments of EEG following or preceding occurrences of a class of discrete events are averaged. The resulting ERP waveform reflects electrical brain activity correlated with the occurrence of members of that class of events. The amplitude, latency, and scalp topography of the various deflections (i.e., components) of the ERP can provide important evidence about the neural mechanisms underlying a variety of cognitive processes (Hillyard \& Picton, 1987; Rugg \& Coles, 1995).

Frenck-Mestre et al. (1997) focused on the N400 component, a negative deflection in the ERP waveform that typically peaks approximately $400 \mathrm{msec}$ after stimulus onset (Kutas \& Hillyard, 1980). The N400 component, likely a complex of related subcomponents (see e.g., Holcomb, Kounios, Anderson, \& West, 1999; Kounios, 1996; Kounios \& Holcomb, 1994), was of particular interest to these investigators, because it has been shown to be sensitive to semantic factors in a variety of tasks (for reviews, see Kounios, 1996; Kutas \& van Petten, 1994; Osterhout \& Holcomb, 1995). Specifically, N400 is typically of larger amplitude to words (or pictures) that do not fit their semantic context (e.g., "He put cream and sugar in his dog/coffee," where dog elicits a larger N400 than does coffee). Although a definitive theory of the N400 does not yet exist, a number of researchers have argued that this component reflects a mechanism that attempts to integrate a piece of information into its semantic context (e.g., Kounios, 1996; Osterhout \& Holcomb, 1995) - hence, the larger amplitude in response to a word such as dog that does not fit well.

Frenck-Mestre et al. (1997) reasoned that if semantic satiation were truly semantic, satiation should diminish the magnitude of the N400 effect (defined as the difference in N400 amplitude to words that fit and do not fit their context). In their task, a category word was presented visually, followed either by the number 3 or by 30 which indicated the number of times the word was to be pronounced. This number was decremented once per second to indicate the rate of pronunciation. Then, following a warning stimulus, another (exemplar) word was presented visually, the participant's task being to judge whether that word was a member of the preceding category. The participants were signaled 1,200 msec after target-word onset to press one of two buttons to indicate this judgment, the 
enforced response delay minimizing the contribution of neural motor activity to the ERP.

Frenck-Mestre et al. (1997) found the standard N400 effect of relatedness-namely, greater negativity (from 300 to $600 \mathrm{msec}$ ) in response to target words that were not drawn from the preceding category than to target words that were drawn from the preceding category. They also found an effect of category repetition (throughout most of the time-course of processing), so that 30 presentations of the category resulted in an ERP to the target that was less negative (i.e., more positive) than when the category had been presented only three times. However, they found no interaction between the effects of relatedness and category repetition on target $\mathrm{N} 400$ amplitude. In other words, prime satiation apparently did not diminish or otherwise modulate the $\mathrm{N} 400$ relatedness effect. Their conclusion was that if the N400 were, in fact, a neural correlate of some aspect of semantic processing, then satiation does not have its effect on any aspect of semantic processing related to the N400.

This surprising result provides a piece to the puzzle of semantic satiation. However, because of the experimental procedure used by Frenck-Mestre et al. (1997), at least one more important piece still remains to be found. Their procedure involved overt verbal repetition of the category prime, followed by category verification of a visually presented target exemplar. This change in stimulus modality from primes to target could be the reason that category repetition did not interact with the effects of categoryexemplar relatedness. Specifically, if there are modalityspecific semantic representation and processing systems, then in the Frenck-Mestre et al. experiment, category repetition could have satiated a semantic system that receives phonological or proprioceptive articulatory inputs and left relatively unaffected another semantic system that receives visual orthographic inputs (and which was subsequently tested). The implication of this analysis is that if the prime and the target had been presented in the same modality, category satiation might have diminished the size of the $\mathrm{N} 400$ relatedness effect, thereby providing evidence that semantic satiation does have an effect at the level of semantic integration. Consistent with this interpretation, Holcomb and Neville $(1990,1991)$ reported that auditory and visual $\mathrm{N} 400 \mathrm{~s}$, although similar in some respects, nevertheless have somewhat different scalp distributions and time-courses, indicating that the neural and cognitive processors associated with the auditory and the visual $\mathrm{N} 400 \mathrm{~s}$ are not identical.

\section{OVERVIEW}

In the present study, the semantic satiation effect was explored with the ERP technique in order to accomplish two goals. First, we sought to examine the neural substrates of semantic satiation when the primes and the target are presented in the same modality. A replication of the Frenck-Mestre et al. (1997) results would then deter- mine that their results were not due to a change of modality and would support their conclusions about a nonsemantic locus for the "semantic" satiation effect. Second, given the findings of Pilotti et al. (1997), it is desirable to manipulate a sensory characteristic of the primes in order to establish more conclusively that the semantic satiation effect is truly semantic in origin, and not simply a byproduct of perceptual adaptation or habituation.

The experimental paradigm adopted here did not require participants to make any overt responses to prime or target words, thereby minimizing the contribution of decision and response processes to target ERPs. The procedure involved the presentation of a continuous stream of words varying in case (Experiment 1) or pitch (Experiment 2B). The task was to view or listen to each word and press a button immediately upon detection of a word that referred to a part of the body (e.g., hand). These body-part words constituted approximately $5 \%$ of all the items and were included only to ensure that the participants would attend to all the words and analyze them for meaning. The primary focus of this study was on the non-body-part words. These consisted of primes and critical items drawn from a variety of semantic categories. A critical word could follow either 1 or 15 presentations of a prime that was either related or unrelated semantically to the critical item. ERPs were averaged separately for critical items following low or high satiation of primes that were related or unrelated to the critical items.

\section{EXPERIMENT 1 Visual Satiation}

Experiment 1 implemented the paradigm just described in a semantic verification task involving visual presentation of repeated primes (in varying case) and critical related/unrelated words. It was predicted that if semantic satiation is indeed semantic, the ERP difference between related and unrelated critical words (i.e., the N400 priming effect) should be significantly smaller following massed presentations of the prime than following a single presentation of the prime.

\section{Method}

Participants. Sixteen student volunteers ( 9 female; mean age, 20.3 years; range, 18-22) served as participants. All were righthanded ( 4 had at least one left-handed relative in the immediate family) and were native speakers of English.

Stimulus Materials and Procedure. The critical word stimuli for this study were formed from 80 triplets of semantically related exemplar words (e.g., dog/cat/pet, truck/car/bus, table/chair/desk). All the words were between three and eight letters in length. The first member of each triplet was designated the prime, whereas the second and third members were designated as critical items. These 80 related triplets were then rearranged to form 80 triplets of semantically unrelated words (e.g., dog/car/table, truck/cat/desk, table/pet/bus). From the related and unrelated triplets above, four stimulus lists were formed so that, within each list, each critical item occurred only once, but, across the four lists, each critical item occurred in each of the four conditions of the experiment (see below). 


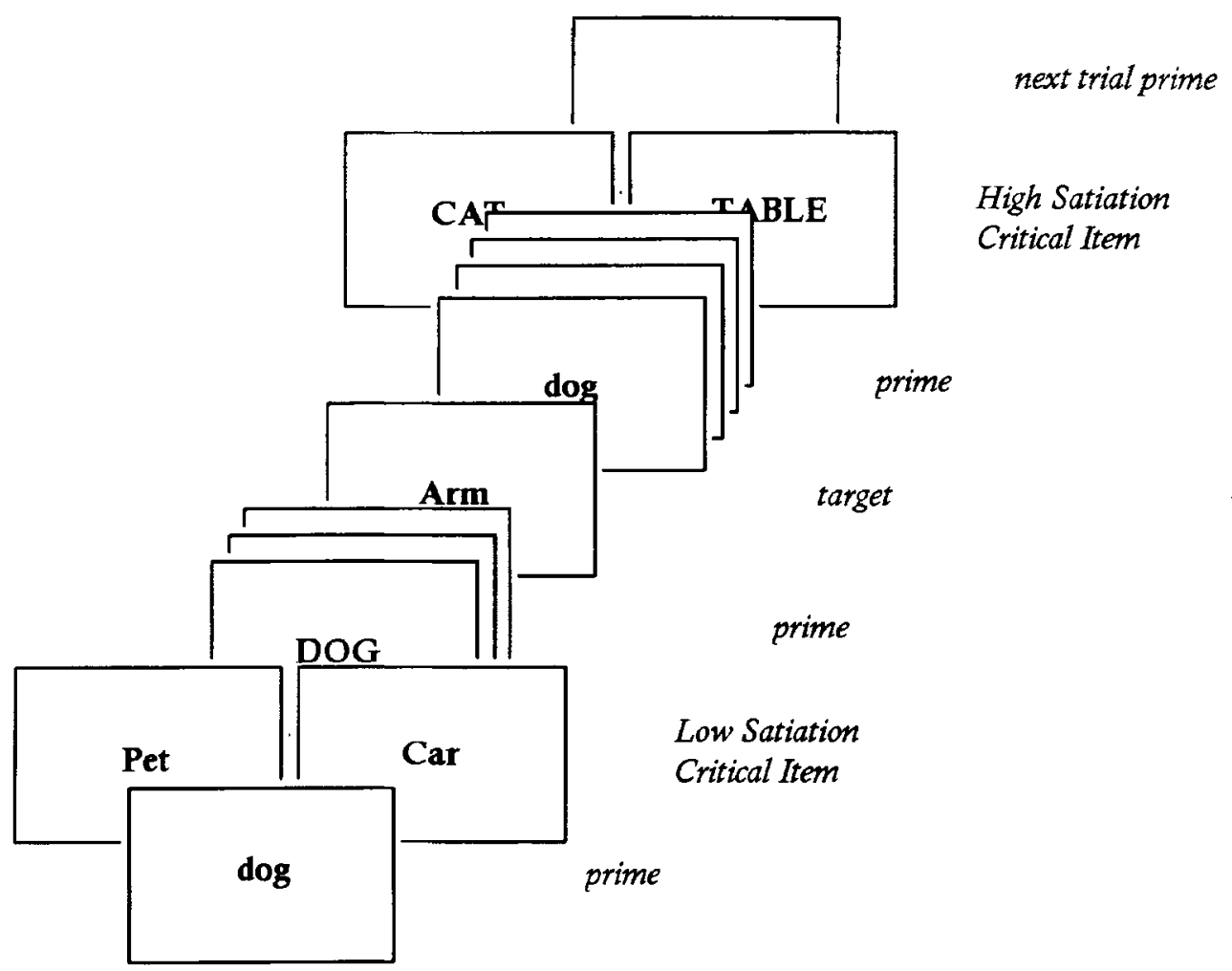

Figure 1. A schematic of a typical trial in Experiment 1. The trial begins with the presentation of the prime word (e.g., dog) in the center of the screen and is followed by either a related or an unrelated lowsatiation critical item (e.g., pet or car). The prime then repeats 14 times with occasional interrupting target items (e.g., arm) and ends with either a related or an unrelated high-satiation critical item (e.g., cat or table).

A trial consisted of 15 presentations of a prime word, with intervening critical words after the 1 st (low satiation) and 15 th repetitions of the prime (high satiation). Fifty percent of the critical words following both low- and high-satiation primes were semantically related to their primes, and $50 \%$ were unrelated. A typical trial proceeded as follows (see Figure 1): (1) A prime word (e.g., dog) was presented for $100 \mathrm{msec}$; (2) $700 \mathrm{msec}$ later, either a semantically related (e.g., cat) or an unrelated (e.g., car) critical word was presented for $100 \mathrm{msec}$; (3) $700 \mathrm{msec}$ later, the prime word was presented again for 100 msec; (4) the prime word was repeated 13 more times (100 msec on, $700 \mathrm{msec}$ off); and (5) $700 \mathrm{msec}$ after the offset of the last repetition of the prime word, a final related (e.g., pet) or unrelated (e.g., table) critical word was presented for $100 \mathrm{msec}$. Seven hundred milliseconds after the offset of the high-satiation critical word, the prime for the next trial was presented.

To help ensure that the participants processed all the words to a relatively deep semantic level, in addition to the critical stimuli listed above, occasional words requiring a behavioral response were randomly interspersed throughout each stimulus list $(n=67$, probability of occurrence on any given trial $=.045)$. These items were all from the category of body parts (e.g., finger, head, hand; note that none of the prime or critical items were from this category). The participants were told to read all of the words presented but to indicate when a word was a body part by rapidly pressing a single button (no response was required for primes and critical items). To help ensure that the participants read each word, especially the repeating prime words, stimulus words randomly changed case between (1) all uppercase letters, (2) all lowercase letters, and (3) the first letter uppercase, with the remaining letters in lowercase (e.g., $D O G$, $\operatorname{dog}, D o g)$. This manipulation was intended to minimize sensory satiation or adaptation to the physical features of the prime and to discourage the participants from using simple physical features to determine that the current item was a repeating prime and not a body part.

An IBM PC AT compatible computer was used for stimulus presentation. All the stimuli were displayed as white letters at the center of a 20 -in. computer monitor and subtended between $0.75^{\circ}$ and $1.75^{\circ}$ of horizontal vertical angle and $0.5^{\circ}$ of vertical visual angle. The participants were run in blocks of 20 trials, punctuated by 2 - to 3-min breaks. A five-trial practice block preceded the actual experiment.

Electroencephalogram recording procedure. The participants were seated in a comfortable chair, and an elastic cap (ElectroCap International, Eaton, $\mathrm{OH}$ ) containing 13 tin electrodes was fitted to their scalp. Four additional electrodes were attached over the left and right mastoids (the right recorded actively, the left serving as a reference for all other sites), below the left eye (for monitoring vertical eye movements and blinks) and to the right of the right eye (for monitoring horizontal eye movements). The scalp sites included seven standard International 10-20 System locations (OI, $\mathrm{O} 2, \mathrm{~F} 7, \mathrm{~F} 8, \mathrm{Pz}, \mathrm{Cz}$, and $\mathrm{Fz}$ ) and six nonstandard locations, including Wernicke's area and its right-hemisphere homologue (30\% of the interaural distance lateral to a point $13 \%$ of the nasion-inion distance posterior to $\mathrm{Cz}$ : WL and WR), left- and right-temporal (33\% of the interaural distance lateral to Cz: TL and TR), and left and right anterior-temporal (one half of the distance between F7 or F8 and T3 or T4: ATL and ATR). All the impedances were maintained below $5 \mathrm{~K}$ ohm. The 16 active electrodes were interfaced to a Grass 
Model 12 amplifier system (bandpass, 0.01-100 Hz; 60- $\mathrm{Hz}$ notch filter), and the EEG was digitized $(200 \mathrm{~Hz}, 12$-bit resolution) continuously on line throughout the experiment by a second IBM PC AT type computer. Averaging was performed off line after the experimental run.

Data analysis. Average ERPs to critical stimuli were formed separately for each of the experimental conditions, which included the two levels of prime satiation (low/high), two critical stimulus types (related/unrelated), and 16 electrode sites, using trials that were free of ocular and amplifier saturation artifact. The ERPs formed in the above manner were digitally low-pass filtered at $20 \mathrm{~Hz}$ and quantified by calculating the mean amplitude in each of three time bands (200-400 msec, $400-600 \mathrm{msec}$, and $600-800 \mathrm{msec}$ ), using the average of the points in the 100 -msec prestimulus period as a baseline.

Each of the three mean amplitude measures were submitted to separate repeated measures analyses of variance (ANOVAs; BMDP2V) employing the correction described by Geisser and Greenhouse (1959) where appropriate. Lateral and midline sites were analyzed in separate ANOVAs. The factors utilized were satiation (high vs. low) and relatedness (related vs. unrelated). For lateralsite analyses, the other factors employed included hemisphere (left vs. right) and anterior-posterior (frontal vs. anterior-temporal vs. temporal vs. temporal-parietal vs. occipital), whereas for the midline-site analyses, there was a single additional factor of anterior-posterior (frontal vs. central vs. parietal).

\section{Results}

Behavioral data. The participants detected words in the body-part category with a mean accuracy of $95 \%$ and a mean reaction time of $653 \mathrm{msec}$.

ERPs: Qualitative characterization. The grand average ERPs to the critical words from 13 scalp sites are plotted in Figures $2 \mathrm{~A}$ (low satiation) and $2 \mathrm{~B}$ (high satiation) for both of the two critical-word types (related and unrelated). As can be seen, the waveforms anterior to the temporal-parietal (Wernicke's) left/right and parietal midline sites are characterized by an early, central anterior negativity (N1) with a peak latency at approximately $120 \mathrm{msec}$. This N1 was followed, at most sites, by a larger positivity (P2) with a peak latency of $200 \mathrm{msec}$ at anterior sites and $220 \mathrm{msec}$ at posterior sites. At the most posterior lateral sites $(\mathrm{O} 1 / 2)$, a somewhat different pattern of early ERP components was apparent. Here, the ERPs started with an early $\mathrm{P} 1$ peaking near $100 \mathrm{msec}$, followed by an N1 with a peak latency near $200 \mathrm{msec}$, a P2 with a peak between 225 and $250 \mathrm{msec}$, and an N2 with a small peak near $300 \mathrm{msec}$ (the latter being especially notable for high-satiation critical items).

At all the sites, there was a relatively large negativegoing component with a peak latency between 300 and $400 \mathrm{msec}(\mathrm{N} 400)$. In the low-satiation condition, the critical-word N400 was followed at anterior sites by a broad, slow, extended negativity that eventually crossed into the positive range near the beginning of the next stimulus epoch ( $800 \mathrm{msec}$ ), at central and temporal sites by a positivity with a peak between 500 and $600 \mathrm{msec}$, and at the most posterior sites by a relatively flat baselinehugging response lasting until the onset of the next word. Following the N400, critical words following high prime satiation produced a positive dip around $500 \mathrm{msec}$ at anterior sites but then went on to produce a substantial neg- ativity near $600 \mathrm{msec}$. This anterior negativity is reminiscent of contingent negative variation (CNV, Grey-Walter, Cooper, Aldridge, McCallum, \& Winter, 1964). At central and temporal sites, critical words following high prime satiation produced a post- $\mathrm{N} 400$ positivity at $500 \mathrm{msec}$ and a subsequent negativity near $600 \mathrm{msec}$. Finally, at the most posterior sites, critical words in the high prime satiation condition produced a large positivity at $500 \mathrm{msec}$, followed by a slow return to baseline near the beginning of the next word's epoch.

Two hundred to $\mathbf{4 0 0}$ milliseconds. The difference between the ERPs to related and unrelated words was not statistically reliable in the $200-400 \mathrm{msec}$ epoch, nor were the interactions of the relatedness factor with the satiation factor, although there was a trend for the unrelated critical items to be more negative than the related critical items in the low-satiation condition and for the reverse to be true for the high-satiation condition [relatedness $\times$ satiation interaction, midline: $F(1,15)=3.67$, $p<.075]$. There was, however, a relatedness $\times$ anteriorposterior interaction at the lateral sites $[F(4,60)=4.44$, $p<.03]$. This was due to the presence of a slightly larger negative-going response for unrelated than for related critical items at the back of the head and for the reverse trend at the front (i.e., related critical items yielding ERPS that were more negative). The only other significant differences in this epoch were that critical words in the lowsatiation condition tended to produce ERPs that were more negative going than critical words in the high-satiation condition across the scalp at lateral sites [i.e., main effect of satiation, lateral: $F(1,15)=5.34, p<.035]$ and at more posterior midline sites [satiation $X$ anterior-posterior interaction: $F(1,15)=7.94, p<.004]$.

Four hundred to 600 milliseconds. Figure 2 indicates that related and unrelated critical items produced their biggest differences in the $400-600 \mathrm{msec}$ epoch [main effect of relatedness, midline: $F(1,15)=7.53, p<.015$; lateral: $F(1,15)=3.93, p<.066$ ] with unrelated critical items producing significantly more negative-going ERPS than did related critical items. Of most interest, however, was the presence of a relatedness $\times$ satiation $\times$ anterior-posterior interaction [midline: $F(2,30)=6.47, p<$ .009 ; lateral: $F(4,60)=3.91, p<.045]$. This interaction indicated that the relatedness effect was larger for the critical words in the low- than in the high-satiation condition, especially at central and anterior sites.

Six hundred to 800 milliseconds. There were no discernable relatedness effects in the 600-800 msec epoch. However, there were differences between ERPs to critical words in the low- and high-satiation conditions, especially at the more anterior sites [midline, satiation $\times$ anterior-posterior interaction: $F(2,30)=23.14, p<$ $.0001]$, with high-satiation critical items producing ERPs that were more negative going.

\section{Discussion}

To summarize the most important results from Experiment 1 , although the relatedness $\times$ satiation interaction 
A
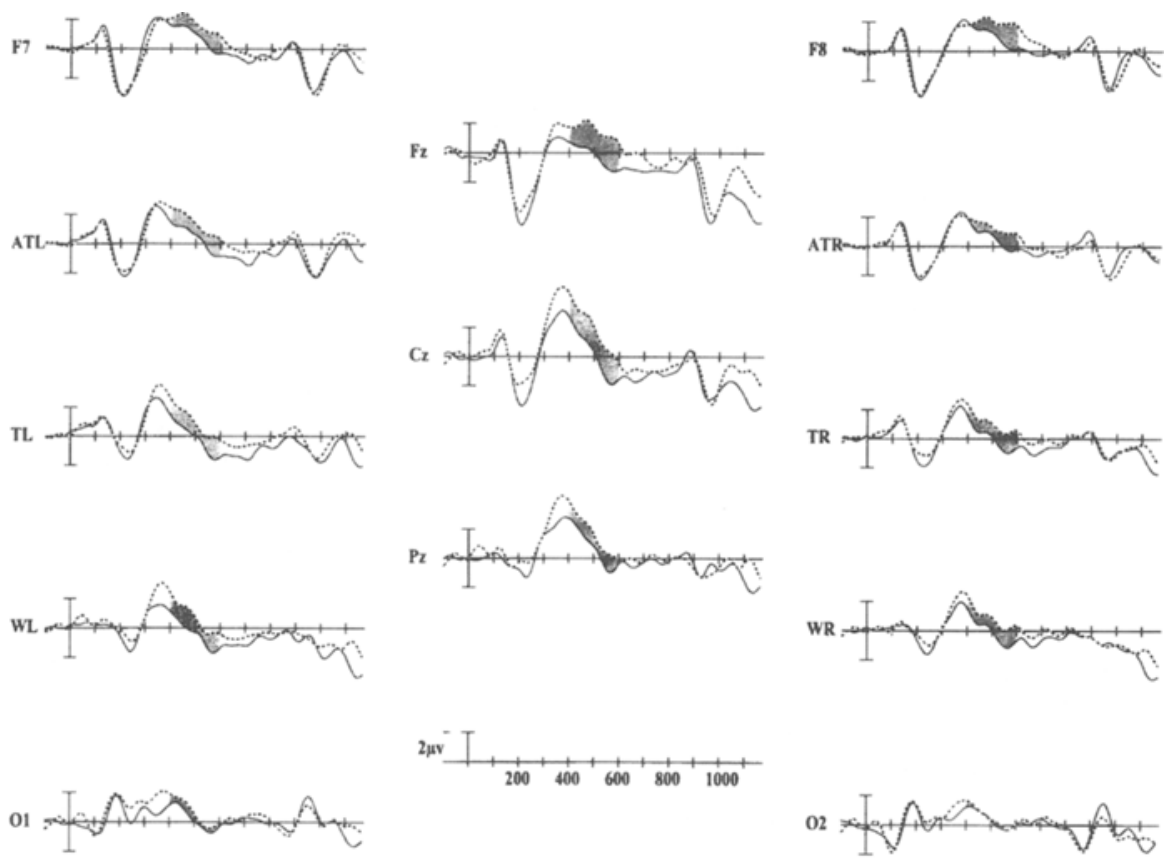

B
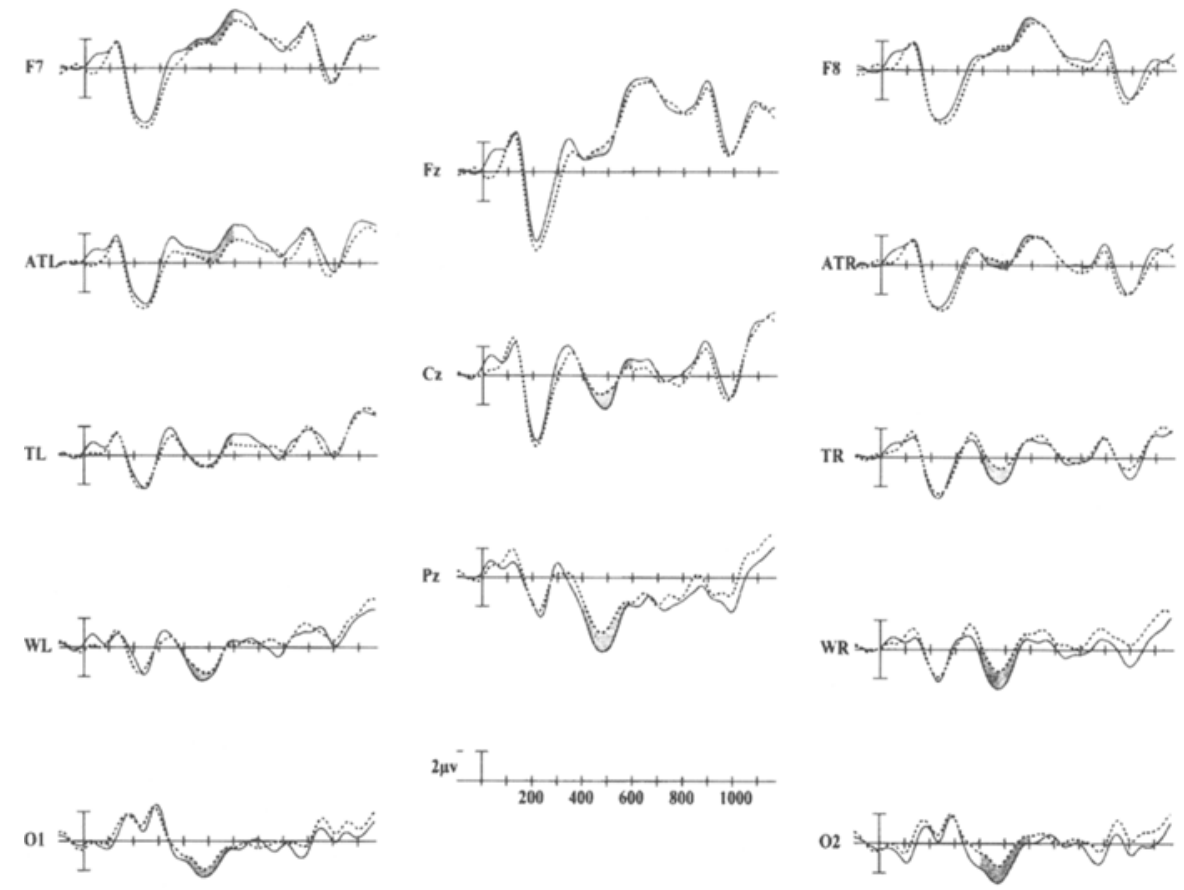

Figure 2. Plotted in this figure are the grand-mean critical-item event-related brain potentials for related critical words (solid lines) and unrelated critical words (dotted lines) for the low-satiation condition (panel $A$ ) and the high-satiation condition (panel B) from the 13 scalp sites of Experiment 1 . Time goes from left to right on the $x$-axes. Stimulus onset is the vertical calibration bar, and the $100-$ msec period prior to this was used as a baseline for the purpose of averaging. The $y$-axis shows voltage, with negative values plotted up (according to ERP convention). Electrode sites at the bottom of the figure are from the back of the head, those at the top of the figure are from the front of the head, those at the right side of the figure are from the right side of the head, and so forth. The difference between the related and the unrelated ERPs for the $400-600 \mathrm{msec}$ time window is shaded. Abbreviations: O1/2, occipital left and right; WL/R, Wernicke's left and right; TL/R, temporal left and right; ATL/R, anterior-temporal left and right; F7/8, frontal left and right; $\mathrm{Pz}$, parietal midline; $\mathrm{Cz}$, central midline (i.e., vertex); $\mathrm{Fz}$, frontal midline. 
achieved only marginal significance in the earliest epoch (200-400 msec), this interaction was significant and more pronounced in the subsequent $400-600 \mathrm{msec}$ epoch that captured most of the N400 activity. This interaction was such that the relatedness effect was larger for critical items following low levels of prime satiation than for those following high levels of prime satiation, meaning that prime satiation decreased the magnitude of the relatedness effect on the critical-item ERP. Moreover, this pattern of results occurred even though the physical features (i.e., case) of the repeated prime varied across presentations, presumably discouraging sensory satiation or adaptation (cf., Pilotti et al., 1997) and encouraging sustained attention to the meaning of each stimulus word, as was required by the experimental paradigm.

The present results also differed from those of FrenckMestre et al. (1997), who found no interaction (i.e., approximate additivity) between the effects of prime satiation and relatedness. This suggests that either the use of spoken primes or the modality shift from primes to critical items incorporated into the design of Frenck-Mestre et al. prevented prime satiation from exerting a discernable effect on semantic processing.

\section{EXPERIMENT 2A Auditory Satiation}

Experiment 2A essentially replicated the design and procedure of Experiment 1, except that the stimuli were presented auditorially. The main issue was whether the interaction between the effects of the relatedness and the satiation factors obtained in Experiment 1 generalizes to the auditory modality. This is important because, as was pointed out above, prior attempts to find satiation with spoken primes have met with mixed success.

\section{Method \\ Participants. Sixteen student volunteers (11 female; mean age, 18.9 years; range, $18-22$ ) participated. All were right-handed ( 7 had at least one left-handed relative in the immediate family) and were native speakers of English. \\ Stimuli and Procedure. The stimuli were the same as those in Experiment 1, with the exception that all were presented binaurally via headphones (Sony model MDR-S30). Prior to the experiment, the words from Experiment 1 were spoken by a female native En- glish speaker, recorded on analog tape, and then digitized at $16 \mathrm{KHz}$ (12-bit resolution, 7-K Hz low-pass filter) on a PC computer. The files containing each word were edited using a visual waveform ed- itor so that the precise onset of the word was aligned with the be- ginning of each file. This ensured accurate time-locking for record- ing ERPs. These files were stored on a stimulus computer and played via a 12-bit D/A converter using the same timing parameters as those used in Experiment 1. Note that although the spoken words differed in their duration (mean, $544 \mathrm{msec}$; minimum, $138 \mathrm{msec}$; maximum, $775 \mathrm{msec}$ ), the time between successive word onsets was the same as that in Experiment 1 (i.e., $800 \mathrm{msec}$ ).}

\section{Results}

Behavioral data. The participants detected words in the body-part category with an accuracy of $93.4 \%$ with a mean reaction time of $754 \mathrm{msec}$.
ERPs: Qualitative characterization. The grandaverage ERPs at 13 scalp sites from Experiment $2 \mathrm{~A}$ are plotted in Figures 3A (low satiation) and 3B (high satiation) for the two types of critical words (related and unrelated). As can be seen, the waveforms anterior to $\mathrm{O} 1 / 2$ in both plots are characterized by an early central-anterior maximum positivity (P1), with a peak latency at approximately $60 \mathrm{msec}$. This P1 was followed, at most sites, by a negative-going wave (N1) with a peak latency near $120 \mathrm{msec}$. The $\mathrm{N} 1$, in turn, was followed by a P2 with a peak latency between $170 \mathrm{msec}$ (low-satiation condition, Figure $3 \mathrm{~A}$ ) and $300 \mathrm{msec}$ (high-satiation condition, Figure $3 \mathrm{~B}$ ). Note that at many sites, the P1-N1-P2 complex stayed entirely on the positive side of the baseline (especially in the low-satiation condition). Also note that just after $800 \mathrm{msec}$, this complex of early components could again be seen, but time-locked to the onset of the next word. At the occipital sites, there was very little activity prior to $200 \mathrm{msec}$.

Following the early components, there was a large negative-going wave that peaked at approximately $400 \mathrm{msec}(\mathrm{N} 400)$ in the low-satiation condition (Figure 3A) and at approximately $500 \mathrm{msec}$ in the high-satiation condition (Figure 3B). As in Experiment 1, the high-satiation condition also produced a much more negative response (CNV) at anterior sites between 400 and $800 \mathrm{msec}$ than occurred in the low-satiation condition. However, in Experiment $2 \mathrm{~A}$, this anterior negativity occurred in the same temporal window as the $\mathrm{N} 400$, instead of in a later temporal window, as occurred for visually presented words in Experiment 1.

Two hundred to $\mathbf{4 0 0}$ milliseconds. In the low-satiation condition, critical words yielded ERPs that tended to be more negative going than those in the high-satiation condition [midline main effect of satiation: $F(1,15)=15.41$, $p<.0014]$, especially over the left hemisphere at lateral sites [satiation $\times$ hemisphere interaction: $F(1,15)=6.67$, $p<.02]$. The critical relatedness $\times$ satiation interaction did not approach significance in this time window (all $F \mathrm{~s}<2.3$ ).

Four hundred to 600 milliseconds. In this epoch, unrelated critical words were more negative going than related ones [main effect of relatedness, midline: $F(1,15)=$ 15.21, $p<.0014$; lateral: $F(1,15)=16.48, p<.001$ ], and this difference tended to be larger over the frontal and central sites along the midline [relatedness $X$ anteriorposterior interaction, midline: $F(2,30)=8.11, p<.0055]$. Critical-word ERPs in the high-satiation condition also tended to be more negative than low-satiation items, but only over the temporal/central/frontal sites [satiation $X$ anterior-posterior interaction, midline: $F(2,30)=37.92$, $p<.00001$; lateral: $F(4,60)=17.53, p<.00001]$. At more posterior sites, high-satiation critical words were actually more positive than low-satiation items, and this trend was larger over the left than over the right hemisphere [satiation $\times$ hemisphere $\times$ anterior-posterior interaction: $F(4,60)=3.85, p<.04$ ]. Finally and most important, the relatedness effect was larger in the low- than in the high-satiation condition [relatedness $\times$ satiation 
A
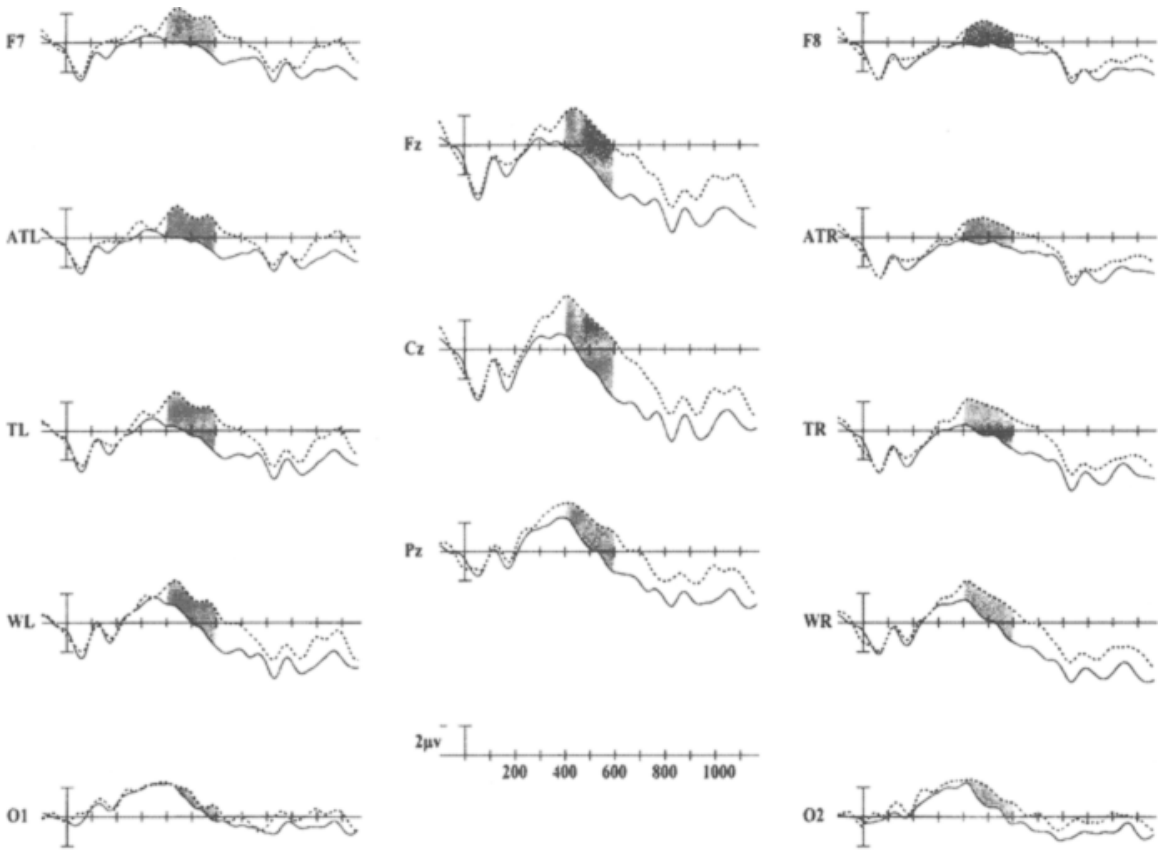

B
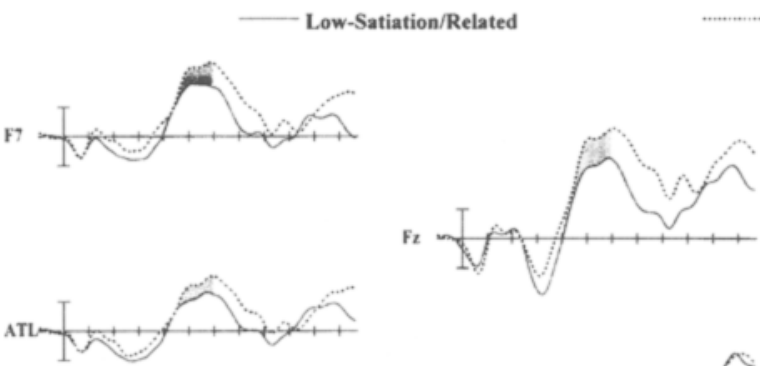

Low-Satiation/Unrelated
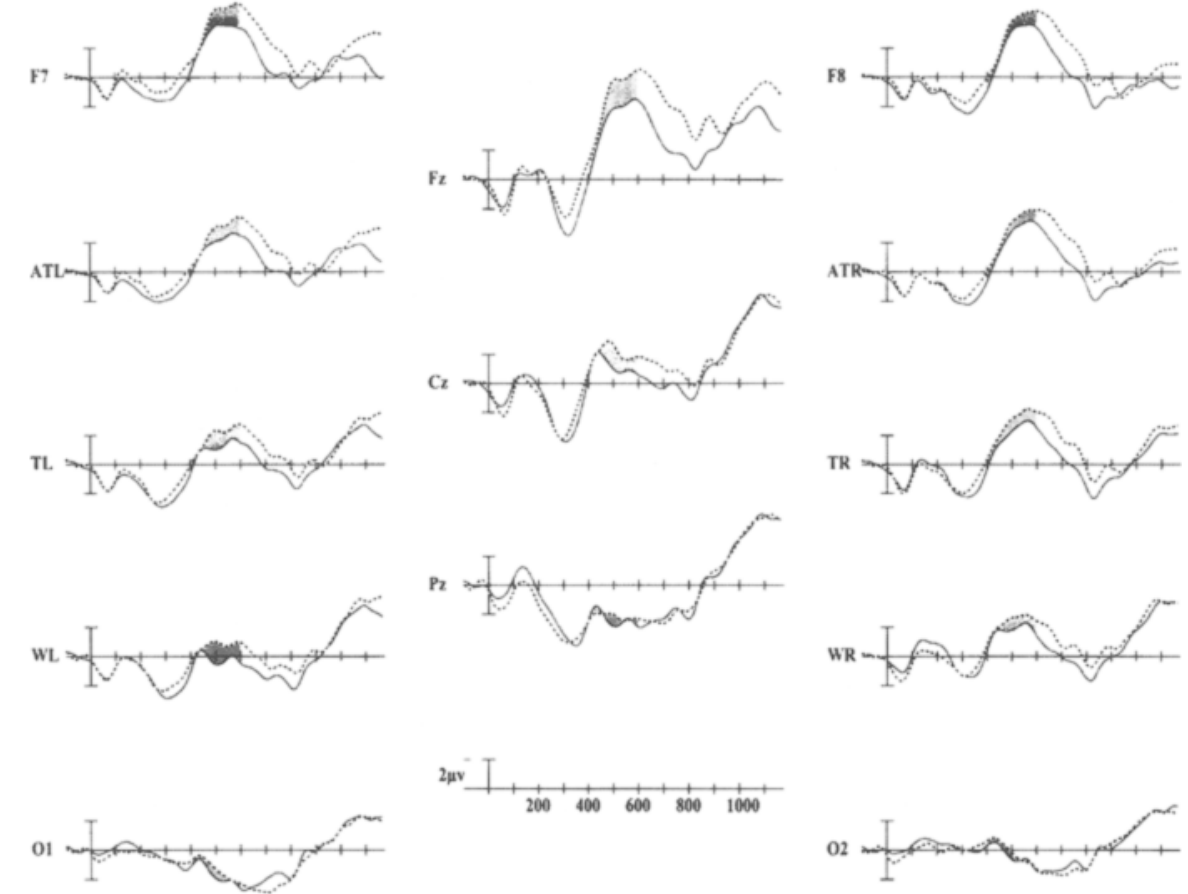

02

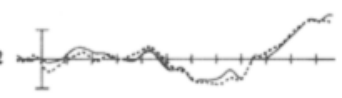

High-Satiation/Related

High-Satiation/Unrelated

Figure 3. Plotted in this figure are the grand-mean critical-item event-related brain potentials (ERPs) for related words (solid) and un related words (dotted) from the low-satiation condition (panel A) and the high-satiation condition (panel B) from the 13 scalp sites of Experiment 2A. Time goes from left to right on the $x$-axes. Stimulus onset is the vertical calibration bar, and the 100-msec period prior to this was used as a baseline for the purpose of averaging. The $y$-axis shows voltage, with negative values plotted up (according to ERP convention). Electrode sites at the bottom of the figure are from the back of the head, those at the top of the figure are from the front of the head, those at the right side of the figure are from the right side of the head, and so forth. The difference between the related and the unrelated ERPs for the $400-600 \mathrm{msec}$ time window is shaded. Abbreviations: O1/2, occipital left and right; WL/R, Wernicke's left and right; TL/R, temporal left and right; ATL/R, anterior-temporal left and right; F7/8, frontal left and right; Pz, parietal midline; $\mathrm{Cz}$, central midline (i.e., vertex); $\mathrm{Fz}$, frontal midline. 
interaction, midline: $F(1,15)=6.96, p<.019$; marginal interaction at the lateral sites: $F(1,15)=3.83, p<.069$ ]. In addition, while the relatedness effect tended to be larger in the low- than in the high-satiation condition at lateral sites, this effect was larger at more temporal-anterior sites over the left hemisphere and at the most posterior sites over the right hemisphere [relatedness $X$ satiation $X$ hemisphere $\times$ anterior-posterior interaction: $F(4,60)=$ $3.43, p<.04]$.

Six hundred to 800 hundred milliseconds. Unrelated critical items continued to be significantly more negative than related critical items [relatedness effect, midline: $F(1,15)=30.17, p<.0001$; lateral: $F(1,15)=$ $19.46, p<.0005]$, and this difference was larger toward the front of the head along the midline [relatedness $x$ anterior-posterior: $F(2,30)=12.46, p<.0006$ ] and at anterior-temporal/temporal/parietal sites in the lateral analyses [relatedness $\times$ anterior-posterior: $F(4,60)=$ $5.44, p<.02]$. Critical words in the high-satiation condition also continued to be more negative than those in the low-satiation condition [main effect of satiation, midline: $F(1,15)=15.18, p<.0014$; lateral: $F(1,15)=11.54, p<$ $.004]$. However, at the parietal-midline and occipitallateral sites, this trend was not apparent. Rather, at these sites, items in the high-satiation condition were actually more positive going than in the low-satiation condition [satiation $\times$ anterior-posterior interaction, midline: $F(2,30)=$ $37.05, p<.00001$; satiation $\times$ anterior-posterior interaction, lateral: $F(4,60)=10.66, p<.0006]$. And finally, the difference in the relatedness effect between the two satiation conditions did not extend to this epoch (i.e., a nonsignificant relatedness $\times$ satiation interaction, $F<1$ ).

\section{Discussion}

The most important result from Experiment 2A was that there was a significant interaction between the relatedness and the satiation factors during the $400-600 \mathrm{msec}$ epoch, although not during the 200-400 and 600$800 \mathrm{msec}$ epochs. More specifically, during the time window encompassing most of the activity of the N400 component, greater prime satiation diminished the magnitude of the relatedness effect on ERP amplitude. Hence, Experiment $2 \mathrm{~A}$ not only replicates this basic finding from Experiment 1, but also demonstrates that it generalizes to the auditory modality.

\section{EXPERIMENT 2B Auditory Satiation With Random Pitch Manipulation}

Experiment 2B was designed to examine the possible role of sensory/acoustic adaptation or satiation in producing semantic satiation (see Balota \& Black, 1997; Pilotti et al., 1997). This was done by means of a slight modification of the design of Experiment 2A, whereby the pitch of the voice speaking the stimulus words was artificially varied from presentation to presentation, sim- ulating changes in the gender or age of the speaker. According to the logic of Pilotti et al., this manipulation should discourage adaptation or satiation at the level of sensory processing. Furthermore, in contrast to the procedure of Pilotti et al., the present procedure also induces participants to attend closely to the meaning of all the words, because a body-part target word could occur at any time in the continuous stream of stimuli.

\section{Method}

Participants. Sixteen student volunteers ( 7 female; mean age, 19 years; range, 18-21) served as subjects. All were right-handed ( 4 had at least one left-handed relative in the immediate family) and were native speakers of English.

Stimuli and Procedure. In Experiment 2B, the stimuli were the same as those in Experiment $2 \mathrm{~A}$, with the exception that repeated prime words were played back to participants at one of three randomly changing frequencies across the 15 presentations of each word: 12,16 , or $20 \mathrm{kHz}$. This manipulation had the effect of changing the perceived gender or age of the speaker from male $(12 \mathrm{kHz})$ to female $(16 \mathrm{kHz})$ to that of a child $(20 \mathrm{kHz})$. For example, if the prime word was $d o g$, a participant might hear the first presentation of $d o g$ in a normal female voice $(16 \mathrm{kHz})$, the second in a male voice $(12 \mathrm{kHz})$, the third in a child's voice $(20 \mathrm{kHz})$, and so forth. Critical words and body-part words were also randomly presented at one of the three frequencies. All else was identical to Experiment $2 \mathrm{~A}$

\section{Results}

Behavioral data. The participants detected words in the body-part category with an accuracy of $96.4 \%$ with a mean reaction time of $744 \mathrm{msec}$.

ERPs: Qualitative characterization. The grand average ERPs from 13 scalp sites in Experiment 2B are plotted in Figures 4A (low satiation) and 4B (high satiation) for both of the two critical-item types (related and unrelated words). As can be seen, the waveforms anterior to $\mathrm{O} 1 / 2$ in both plots are characterized by an early, central-anterior maximum positivity ( $\mathrm{P} 1)$ with a peak latency at approximately $60 \mathrm{msec}$. This $\mathrm{P} 1$ was followed, at most sites, by a negative-going wave $(\mathrm{N} 1)$ with a peak latency near $120 \mathrm{msec}$. The N1, in turn, was followed by a P2 with a peak latency between $170 \mathrm{msec}$ (low-satiation condition, Figure 4A) and $250 \mathrm{msec}$ (high-satiation condition, Figure 4B). Note that at many sites, the P1-N1-P2 complex stayed entirely on the positive side of the baseline, especially in the low-satiation condition. Also note that just after $800 \mathrm{msec}$, this complex of early components could be seen again, but time-locked to the onset of the next word. At the occipital sites, there was very little activity prior to $200 \mathrm{msec}$.

Following the early components, there was a large negative-going wave that peaked near $400 \mathrm{msec}(\mathrm{N} 400)$ in the low-satiation condition (Figure 4A) and between 500 and $600 \mathrm{msec}$ in the high-satiation condition (Figure 4B). Similar to their visual counterparts (Experiment 1 ), the high-satiation condition also yielded a much more negative response (CNV) at anterior sites between 400 and 800 msec than did the low-satiation condition. 
A
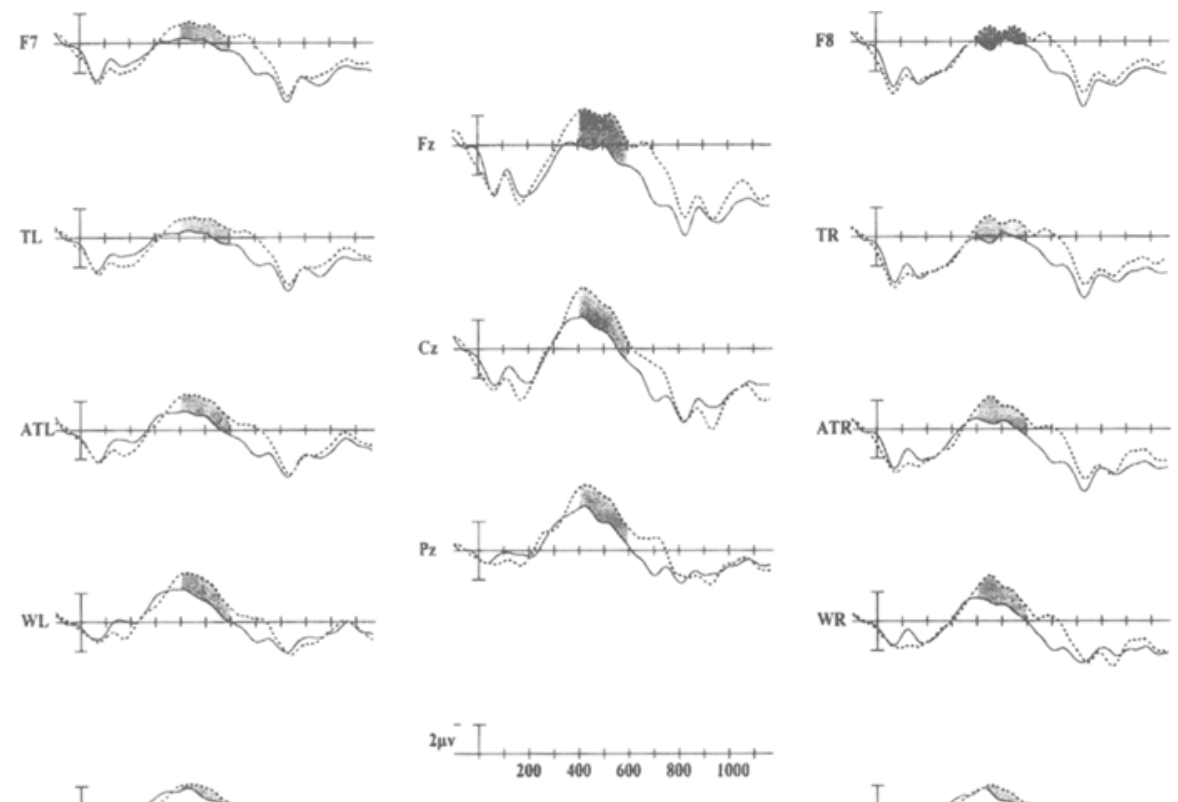
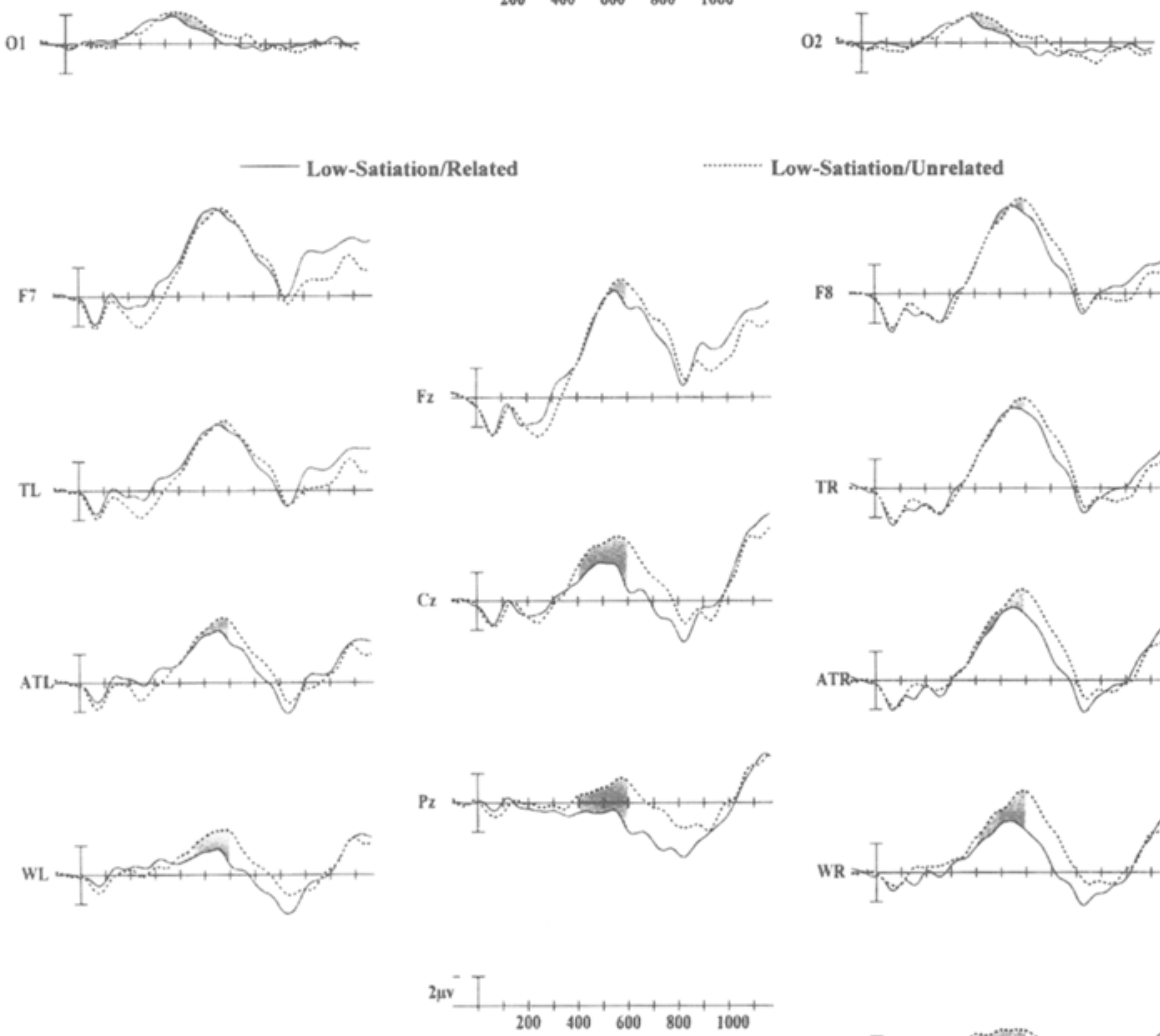

Low-Satiation/Unrelated

B
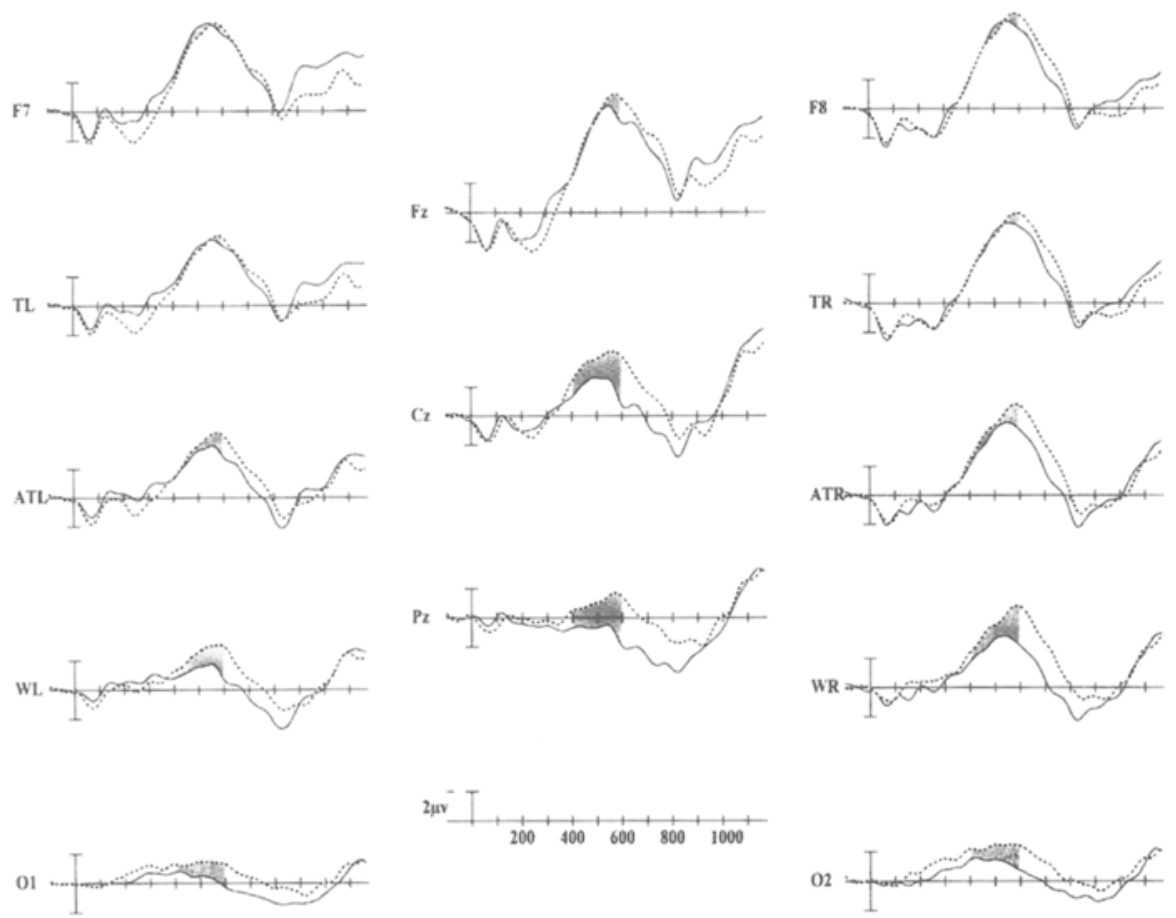

Figure 4. Plotted in this figure are the grand-mean critical-item event-related brain potentials (ERPs) for related words (solid) and unrelated words (dotted) from the low-satiation condition (panel A) and the high-satiation condition (panel B) from the 13 scalp sites of Experiment 2B. Time goes from left to right on the $x$-axes. Stimulus onset is the vertical calibration bar, and the 100-msec period prior to this was used as a baseline for the purpose of averaging. The $y$-axis shows voltage, with negative values plotted up (according to ERP convention). Electrode sites at the bottom of the figure are from the back of the head, those at the top of the figure are from the front of the head, those at the right side of the figure are from the right side of the head, and so forth. The difference between the related and the unrelated ERPs for the $400-600$ msec time window is shaded. Abbreviations: O1/2, occipital left and right; WL/R, Wernicke's left and right; TL/R, temporal left and right; $A T L / R$, anterior-temporal left and right; F $7 / 8$, frontal left and right; Pz, parietal midline; $\mathrm{Cz}$, central midline (i.e., vertex); $\mathrm{Fz}$, frontal midline. 
However, as in Experiment 2A, in Experiment 2B this anterior negativity occurred in the same temporal window as the $\mathrm{N} 400$ (instead of a later one for visual words).

Two hundred to $\mathbf{4 0 0}$ milliseconds. Critical items in the low-satiation condition tended to be more negative going than those in the high-satiation condition, but only at more anterior sites [satiation $\times$ anterior-posterior interaction, midline: $F(1,15)=5.82, p<.02]$, and only over the left hemisphere [satiation $\times$ hemisphere interaction: $F(1,15)=4.61, p<.05$ ]. At the most posterior midline site and at right-hemisphere lateral sites, low-satiation critical words actually yielded a more positive waveform than occurred for high-satiation items. The critical relatedness $\times$ satiation interaction did not approach significance (all $F \mathrm{~s}<1.65$ ).

Four hundred to 600 milliseconds. In this epoch, unrelated critical items were more negative going than related items [main effect of relatedness, midline: $F(1,15)=$ 18.87, $p<.0006$; lateral: $F(1,15)=9.07, p<.009]$ ] Unlike Experiment $2 \mathrm{~A}$, this effect did not vary significantly across the scalp. Critical words in the high-satiation condition also tended to be more negative than those in the low-satiation condition, especially over the temporal/ central/frontal sites [satiation $\times$ anterior-posterior, midline: $F(2,30)=38.17, p<.00001$; lateral: $F(4,60)=13.81$, $p<.0006]$. At more posterior sites, critical items in the high-satiation condition were actually more positive than those in the low-satiation condition, but only over the left hemisphere [satiation $\times$ hemisphere $\times$ anterior-posterior interaction: $F(4,60)=3.44, p<.04]$. Finally, the relatedness effect was larger for low- than for high-satiation critical words, but only over more anterior sites [relatedness $\times$ satiation $\times$ anterior-posterior interaction, midline: $F(2,30)=9.12, p<.002$; marginally significant interaction at lateral sites: $F(4,60)=3.29, p<.07]$. Note that in Figure $4 \mathrm{~B}$, there was very little evidence of a relatedness effect in the high-satiation condition at anterior sites (i.e., as compared with the low-satiation condition, Figure 4A).

Six hundred to 800 milliseconds. Unrelated critical words continued to be significantly more negative than related words [main effect of relatedness, midline: $F(1,15)=14.78, p<.0016$; lateral: $F(1,15)=35.57, p<$ .00001 . High-satiation critical words also continued to be more negative than low-satiation ones [main effect of satiation, midline: $F(1,15)=8.42, p<.011$; lateral: $F(1,15)=13.46, p<.0023]$. However, at the most posterior sites (e.g., the occipital) this latter trend was not apparent. For example, as in Experiment 2A, at the midline-parietal site, high-satiation items were actually more positive going than low-satiation ones [satiation $x$ anterior-posterior interaction, midline: $F(2,30)=64.69$, $p<.00001$; satiation $\times$ anterior-posterior interaction, lateral: $F(4,60)=11.78, p<.0007]$. Most important, the difference in the relatedness effect for low- and high-satiation critical words was not significant (relatedness $\times$ satiation interaction, $F \mathrm{~s}<1$ ).

\section{Discussion}

Experiment $2 \mathrm{~B}$ yielded an interaction between satiation and relatedness (and a topographic factor) during both the 200-400 and the 400-600 msec time windows, the latter capturing most of the activity of the N400 component. This replicates the central result from Experiments 1 and $2 \mathrm{~A}$. Furthermore, Experiment $2 \mathrm{~B}$ yielded this result despite the fact that an important sensory characteristic of the stimuli (i.e., pitch) was randomly manipulated. This supports the notion that semantic satiation is not merely a by-product of sensory satiation or adaptation.

\section{GENERAL DISCUSSION}

The present study focused on neurophysiological correlates of semantic satiation, with the goal of determining whether such satiation can be linked with processes known to be associated with semantic information processing. In particular, the aims were (1) to ascertain whether prime satiation influences the effect of relatedness on ERPs, particularly the N400, an ERP component understood to play a significant role in semantic processing (see Frenck-Mestre et al., 1997), and (2) to ascertain whether such effects on the $\mathrm{N} 400$ are a mere by-product of sensory satiation/adaptation (consistent with Pilotti et al., 1997) or whether they result from direct effects of prime satiation on downstream semantic processing (as was argued by Balota \& Black, 1997).

Experiments 1,2A, and $2 \mathrm{~B}$ all found interacting effects of prime satiation and relatedness on ERP amplitude, particularly in the N400 time window. Moreover, these effects were found both when the primes and critical items were presented in the visual modality (Experiment 1) and when the primes and critical items were presented in the auditory modality (Experiments $2 \mathrm{~A}$ and $2 \mathrm{~B}$ ). These results contrast with those of Frenck-Mestre et al. (1997), who found approximately additive effects of prime satiation and prime-target relatedness on ERP amplitude in the N400 time window when the primes and the critical items were presented in different modalities. Overall, the existence of within-modality satiation effects on the $\mathrm{N} 400$, coupled with the previous failure to find such effects in a cross-modality paradigm (Frenck-Mestre et al., 1997), suggests the existence of modality-specific semantic representation or processing systems that can be selectively influenced.

The other major finding was that there were still interacting effects of satiation and relatedness on ERP amplitude in the $\mathrm{N} 400$ epoch when the primes were presented visually in different cases (Experiment 1 ) and when the primes were presented auditorially at different pitches (Experiment 2B), these manipulations being intended to minimize sensory satiation as a cause of downstream effects on semantic processing. These findings support the notion that semantic satiation is not a mere by-product of sensory or perceptual adaptation. These results agree with Balota and Black's (1997) conclusion that semantic satia- 
tion is not phonological in nature, although they disagree with the findings of Pilotti et al. (1997), who found obliteration of behavioral semantic satiation effects when the primes were presented in a variety of voices. However, as was explained in the introduction, neither of the latter two behavioral studies should be taken as definitive by themselves, both because behavioral concomitants of semantic satiation have proven to be fragile and because specific features of their experimental procedures and designs may have limited the sensitivity and generality of their experiments. Because the present study rests on this prior foundation, it sidesteps the problematic aspects delineated above. Nevertheless, it should be kept in mind that the conclusion that satiation can occur at the semantic level of processing does not imply that semantic processing cannot be impaired by satiation or adaptation of upstream perceptual processes.

A final question concerns how the present data can be reconciled with the view that the N400 reflects a semantic integration mechanism. Specifically, if semantic satiation reduces the magnitude of the N400 effect and if the N400 reflects a semantic integration mechanism, does this imply that satiation actually reduces the amount of work necessary to integrate a critical item into its preceding semantic context? In other words, do these data imply that satiation makes it easier (rather than more difficult) to process the critical item? Not necessarily, since there is an alternate interpretation that is more consistent with the notion that semantic satiation inflicts a cost on processing. In particular, the present results could simply mean that less integrative activity occurs after satiation, possibly because satiation causes refractoriness or adaptation in the semantic system, thereby rendering it temporarily less responsive, perhaps by weakening the context into which the critical item is to be integrated. So, if there is little or no semantic context remaining after satiation, there will not be much integrative activity; hence, there will be a smaller N400 effect. This weakening of the semantic context with repetition may be analogous to a stabilized retinal image's seeming to disappear.

In conclusion, the present results support the notion that semantic satiation is, in fact, semantic in nature, rather than having a primarily sensory or perceptual locus. Furthermore, semantic satiation appears to occur within modality-specific semantic systems, rather than in an amodal representation or processing system.

\section{REFERENCES}

Balota, D. A., \& Black, S. (1997). Semantic satiation in healthy young and older adults. Memory \& Cognition, 25, 190-202.
Frenck-Mestre, C., Besson, M., \& Pynte, J. (1997). Finding the locus of semantic satiation: An electrophysiological attempt. Brain \& Language, 57, 406-422.

Geisser, S., \& Greenhouse, S. (1959). On methods in the analysis of profile data. Psychometrika, 24, 95-112.

Grey-Walter, W., CoOPER, R., Aldridge, V. J., MCCallum, W. C., \& WINTER, A. L. (1964). Contingent negative variation: An electric sign of sensorimotor association and expectancy in the human brain. Nature, 203, 380-384.

Hillyard, S. A., \& Picton, T. W. (1987). Electrophysiology of cognition. In F. Plum (Ed.), Handbook of physiology: Sec. I. Neurophysiology (pp. 519-584). New York: American Physiological Society.

Holcomb, P. J., Kounios, J., ANDERSON, J. E., \& West, W. C. (1999). Dual coding, context availability, and concreteness effects in sentence comprehension: An electrophysiological investigation. Journal of Experimental Psychology: Learning, Memory, \& Cognition, 25, $721-742$.

Holcomb, P. J., \& Neville, H. (1990). Semantic priming in visual and auditory lexical decision: A between modality comparison. Language \& Cognitive Processes, 5, 281-312.

Holcomb. P. J., \& Neville, H. J. (1991). The electrophysiology of spoken sentence processing. Psychobiology, 19, 286-300.

KouNIOS, J. (1996). On the continuity of thought and the representation of knowledge: Electrophysiological and behavioral time-course measures reveal levels of structure in semantic memory. Psychonomic Bulletin \& Review, 3, 265-286.

Kounjos, J., \& HolcomB. P. J. (1994). Concreteness effects in semantic processing: ERP evidence supporting dual-coding theory. Journal of Experimental Psychology: Learning. Memory, \& Cognition, 20, 804-823.

KuTAS, M., \& Hillyard, S. A. (1980). Reading senseless sentences: Brain potentials reflect semantic incongruity. Science, 207, 203-205.

Kutas, M., \& van Petten, C. (1994). Psycholinguistics electrified. In M. Gernsbacher (Ed.), Handbook of psycholinguistics (pp. 84-143). New York: Academic Press

OSterhout, L., \& Holcomb, P. J. (1995). Event-related potentials and language comprehension. In M. Rugg \& M. G. H. Coles (Eds.), Electrophysiology of mind (pp. 171-215). Oxford: Oxford University Press.

Pilotti. M., Antrobus, J. S., \& Duff, M. (1997). The effect of presemantic acoustic adaptation on semantic "satiation." Memory \& Cognition, 25, 305-312.

RugG, M., \& Coles, M. G. H. (1995). Electrophysiology of mind. Oxford: Oxford University Press.

Severance, E., \& Washburn, M. F. (1907). The loss of associative power in words after long fixation. American Journal of Pychology, 18, $182-186$.

SMITH, L. C. (1984). Semantic satiation affects category membership decision time but not lexical priming. Memory \& Cognition, 12, 483488.

Smith. L. C., \& Klein. R. (1990). Evidence for semantic satiation: Repeating a category slows subsequent semantic processing. Journal of Experimental Psychology: Learning, Memory. \& Cognition, 16, 852861 .
(Manuscript received January 13, 1999; revision accepted for publication October 18, 1999.) 